\section{The African Elephant Database}

As the members of the IUCN/SSC African Elephant Specialist Group's (AfESG) Data Review Task Force and co-authors of the African Elephant Database (AED) reviewed by Sharp (2000), we would like to address Mr Sharp's two main criticisms.

The aim of the AED is to present objective information on the status of the African elephant at the continental level with an indication of the quality of this information. Estimates of elephant numbers are contributed by a wide range of individuals, government management authorities and non-governmental organizations. In practice, it proved difficult to put this information together into a meaningful overall result and Mr Sharp criticises our seemingly idiosyncratic way of reporting the estimates.

The quality of population estimates reported in the AED varies widely; a high proportion of the estimates are simply guesses and many come without an estimate of statistical error. Combining them to obtain a mean and combined error estimate is neither possible nor valid. It is because of this that we categorize estimates into the classes that Mr Sharp dislikes. The system that we adopted, after much discussion, still makes a useful statement about the range of possible values for the elephant populations without attempting to 'fudge' a confidence interval. It also focuses attention on a minimum known population, the 'definites', rather than a central value, which is a sum of the estimates. To have estimated otherwise would imply that the different types of estimates are of equal reliability. This would have been absurd given the differing biases of the estimates, the guesses, and the gaps in coverage.

$\mathrm{Mr}$ Sharp's second criticism is that we have not reported on trends in African elephant populations. Like many readers he expects that, in addition to merely collecting and reporting the available information, the AED should provide further interpretation. In order to detect trends, data must be collected repeatedly and in a comparable manner each time. However, there are only a few populations of African elephants that satisfy these conditions. These can tell us little about continental trends because the well-surveyed populations are mainly from the savannah areas of southern and eastern Africa. There are still relatively unknown, and possibly large, populations of elephants in the forests of western and central Africa. Trends in these forest populations will not necessarily mirror those in the savannah populations. While trends in individual populations may be interesting, the goal of the AED remains to provide a continental overview.

The compilation of all the available information on the status of the best-studied mammal on the continent shows that there are still great gaps in our knowledge. This is perhaps the most important conclusion that can be drawn from the AED. The lack of good information should, through the medium of the AED, be a catalyst for the improvement of survey standards. Consequently we are working, through the AfESG membership, government agencies, NGOs and individuals involved in survey work, to improve the standards of elephant counting across the range of the African elephant, to stimulate the counting of elephant populations that have never been surveyed, and the recounting of those that have not been surveyed for many years. Under this stimulus, methods, data quality and coverage are improving. With such improvements we may indeed soon be able to use the AED to tackle the issue of trends.

Richard F.W. Barnes

G. Colin Craig

Holly T. Dublin

Chris R. Thouless

Members of the AfESG'S Data Review Task Force, c/o AfESG Secretariat, PO Box 62440, Nairobi, Kenya

\section{References}

Sharp, R. (2000) Review of the African elephant database 1999. Oryx, 34, 346.

\section{A reply to the response by Holt, Papastavrou \& Phillips to CITES Conference of Parties 11: an FFI perspective}

Holt et al. (2000) criticise the reference, in my news report of the recent CITES Conference of Parties (CoP) 11 in Nairobi (Sharp, 2000), to minke whale populations having recovered and the International Whaling Commission (IWC) having declined to follow the advice of its Scientific Committee on the matter. They challenge me to produce references.

Manifestly I was not writing a scientific peer-reviewed article on whaling, but giving an overview of the meeting from an FFI perspective. It would not be practicable to discuss all the distinct minke whale 